\title{
Transforms and Operators for Directional Bioimage Analysis: A Survey
}

\author{
Zsuzsanna Püspöki, Martin Storath, Daniel Sage, and Michael Unser * \\ Biomedical Imaging Group, École polytechnique fédérale de Lausanne (EPFL), Station 17, \\ CH-1015 Lausanne VD, Switzerland
}

Summary. We give a methodology-oriented perspective on directional image analysis and rotation-invariant processing. We review the state of the art in the field and make connections with recent mathematical developments in functional analysis and wavelet theory. We unify our perspective within a common framework using operators. The intent is to provide image-processing methods that can be deployed in algorithms that analyze biomedical images with improved rotation invariance and high directional sensitivity. We start our survey with classical methods such as directional-gradient and the structure tensor. Then, we discuss how these methods can be improved with respect to robustness, invariance to geometric transformations (with a particular interest in scaling), and computation cost. To address robustness against noise, we move forward to higher degrees of directional selectivity and discuss Hessian-based detection schemes. To present multiscale approaches, we explain the differences between Fourier filters, directional wavelets, curvelets, and shearlets. To reduce the computational cost, we address the problem of matching directional patterns by proposing steerable filters, where one might perform arbitrary rotations and optimizations without discretizing the orientation. We define the property of steerability and give an introduction to the design of steerable filters. We cover the spectrum from simple steerable filters through pyramid schemes up to steerable wavelets. We also present illustrations on the design of steerable wavelets and their application to pattern recognition.

\section{Introduction}

Directionality and orientation information is very useful for the quantitative analysis of images. By those terms, we refer to local directional cues and features that one can identify in natural images. The area of applications based on the detection of orientation is continuously growing as the importance of directionality is becoming more and more relevant in image processing. The range of applications spans topics from astronomy (Bernasconi et al, 2005; Schuh et al, 2014; Yuan et al, 2011), aerial and satellite imagery (Jiuxiang et al, 2007; Tupin et al, 1998), material sciences (Dan et al, 2012), to biological and medical applications. Focusing on the last two categories, the palette is quite broad: detection of nodules in the lungs (Agam et al, 2005) and vessels in retinal fundus images (Lam et al, 2010; Patton et al, 2006), bioimaging (Honnorat et al, 2011), neuroimaging (Gonzalez et al, 2009; Meijering et al, 2004). Investigations of collagen in the arterial adventitia also rely on directional analysis (Rezakhaniha et al, 2012). Neuron tracking is of primal importance to

*This work was partially supported by the ERC (ERC-2010-AdG 267439-FUN-SP), the Hasler Foundation, and the Swiss National Foundation (grant number: 200020-162343). 
understand the development of the brain and requires robust directional image-analysis tools to capture dendrites in 2D and 3D (Meijering, 2010). In (Jacob et al, 2006), the authors used steerable ridge detector (based on (Canny, 1986)) to study the aging of elastin in human cerebral arteries. In (Aguet et al, 2009), 3D steerable filters were applied to the estimation of orientation and localization of fluorescent dipoles.

Researchers in image analysis are getting inspiration from the human visual system. In the early 1960s, it was demonstrated that directionality plays a key role in visual perception: The neurophysiological findings of Huber and Wiesel initiated a field of research for decades to come (Hubel and Wiesel, 1962). Follow-up studies confirmed that the organization of the primary visual cortex makes our visual perception particularly sensitive to the directional clues carried by edges, ridges, and corners (Marr and Hildreth, 1980; Olshausen and Field, 1996). Our visual system is able to efficiently capture and summarize this information using a small number of neuronal cells.

Based on these structures, many image-analysis methods have been proposed, but they face several challenges. One of them is efficiency with respect to computational resources, because real-time applications and the processing of large multidimensional data (e.g., multichannel time-lapse sequences of images or volumes) demand fast algorithms. Another challenge is to design algorithmic detectors of orientation that acknowledge that patterns in natural images usually have an unknown size and location. Robustness to noise is another desirable trait.

This survey aims at providing the reader with a broad overview of techniques for the directional analysis of images. It is intended to be used as a guide to state of the art methods and techniques in the field. In this paper, we focus on the applications in bioimaging, presenting and comparing the described methods on experimental data.

We focus on the continuous domain setup for explaining the relevant concepts because it allows for convenient, compact, and intuitive formulation. It primarily involves differential and convolution operators (smoothing filters and wavelets) that are acting on continuously defined images, $f(\boldsymbol{x}), \boldsymbol{x}=\left(x_{1}, x_{2}\right) \in \mathbb{R}^{2}$. The final transcription of a continuous domain formula into an algorithm requires the discretisation of the underlying filters which can be achieved using standard techniques. For instance, partial derivatives can be closely approximated using finite differences, while there are well-established techniques for computing wavelets using digital filters. For further implementation details, we are giving pointers to the specialised literature.

\section{Derivative-Based Approaches}

\subsection{Gradient Information and Directional Derivatives}

Some of the earliest and simplest techniques in image analysis to account for orientation rely on gradient information. Intuitively, the direction of the gradient corresponds to the direction of steepest ascent. The local direction of an image $f$ at $\boldsymbol{x}_{0}$ can be estimated in terms of the direction orthogonal to its gradient. A direction is specified in $\mathbb{R}^{2}$ by a unit vector $\boldsymbol{u}=\left(u_{1}, u_{2}\right) \in \mathbb{R}^{2}$ with $\|\boldsymbol{u}\|=1$. The first-order directional derivative $\mathrm{D}_{\boldsymbol{u}} f$ along the direction $\boldsymbol{u}$ can be expressed in terms of the gradient

$$
\mathrm{D}_{\boldsymbol{u}} f\left(\boldsymbol{x}_{0}\right)=\lim _{h \rightarrow 0} \frac{f\left(\boldsymbol{x}_{0}\right)-f\left(\boldsymbol{x}_{0}-h \boldsymbol{u}\right)}{h}=\left\langle\boldsymbol{u}, \boldsymbol{\nabla} f\left(\boldsymbol{x}_{0}\right)\right\rangle,
$$



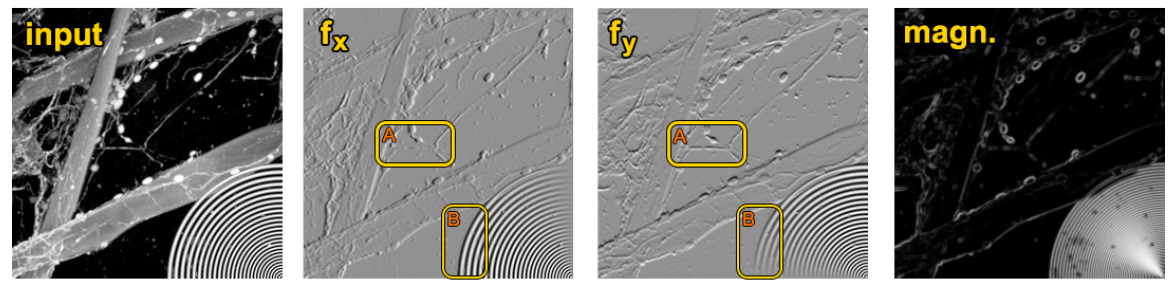

Fig. 1. Illustration of the use of gradient operators, from left to right: (1) Input image: confocal micrograph showing nerve cells growing along fibers made from a specially modified silk that is similar to that made by spiders and silkworms. This input image is from the Cell Image Library (http://www.cellimagelibrary.org/images/38921). Right lower corner: bright arcs with different scales, artificially added. (2) the $x_{1}$ (or horizontal) component of the gradient is the directional derivative along $\boldsymbol{u}=(1,0)$. (3) the $x_{2}$ (or vertical) component of the gradient is the directional derivative along $\boldsymbol{u}=(0,1)$. (4) Magnitude of the gradient vector. Highlighted window A: Horizontal edges are attenuated in case of directional derivative along $\boldsymbol{u}=(1,0)$ and enhanced/kept in case of directional derivative along $\boldsymbol{u}=(0,1)$. Highlighted window B: Vertical edges are attenuated in case of directional derivative along $\boldsymbol{u}=(0,1)$ and enhanced/kept in case of directional derivative along $\boldsymbol{u}=(1,0)$. All the images were produced by the ImageJ/Fiji plugin OrientationJ.

where the right hand side is the inner product between $\boldsymbol{u}$ and the gradient vector $\boldsymbol{\nabla} f\left(\boldsymbol{x}_{0}\right)$ evaluated at $x_{0}$. We note that (1) is maximum when $\boldsymbol{u}$ is collinear to $\nabla f\left(\boldsymbol{x}_{0}\right)$ (by the Cauchy - Schwartz inequality). Conversely, $\mathrm{D}_{\boldsymbol{u}_{0}} f\left(\boldsymbol{x}_{0}\right)$ vanishes when $\boldsymbol{u}_{0} \perp \nabla f$, so that $\boldsymbol{u}_{0}$ provides us with a local estimate of the directionality of the image. Figure 1 illustrates the application of the gradient operators.

Gradient-based orientation estimators are frequently used as they can be discretized and implemented easily. However, the gradient-based estimation of the orientations is sensitive to noise. The robustness can be improved by smoothing the image by a Gaussian kernel before taking the derivative. A still very popular method based on gradients is Canny's classical edge detector (Canny, 1986).

\subsection{Improving Robustness by the Structure Tensor}

The estimation of the local orientation using derivatives can be made more robust by using the structure tensor (Jahne, 1997). The structure tensor is a matrix derived from the gradient of the image and can be interpreted as a localized covariance matrix of the gradient. Since the pioneering work of Förstner (Förstner, 1986), Bigün (Bigun, 1987), and Harris and Stephens (Harris and Stephens, 1988), the structure tensor has become a tool for the analysis of low-level features, in particular for corner and edge detection as well as texture analysis. In $2 \mathrm{D}$, the structure tensor at location $\boldsymbol{x}_{0}$ is defined by

$$
\boldsymbol{J}\left(\boldsymbol{x}_{0}\right)=\int_{\mathbb{R}^{2}} w\left(\boldsymbol{x}-\boldsymbol{x}_{0}\right)(\boldsymbol{\nabla} f(\boldsymbol{x})) \boldsymbol{\nabla}^{T} f(\boldsymbol{x}) \mathrm{d} x_{1} \mathrm{~d} x_{2},
$$

where $w$ is a nonnegative isotropic observation window (e.g., a Gaussian) centered at $\boldsymbol{x}_{0}$. More explicitly, the $(2 \times 2)$ matrix $J\left(x_{0}\right)$ reads 


$$
\begin{aligned}
\boldsymbol{J}\left(\boldsymbol{x}_{0}\right) & =\int_{\mathbb{R}^{2}} w\left(\boldsymbol{x}-\boldsymbol{x}_{0}\right)\left(\begin{array}{cc}
f_{x_{1}}^{2}(\boldsymbol{x}) & f_{x_{1}}(\boldsymbol{x}) f_{x_{2}}(\boldsymbol{x}) \\
f_{x_{2}}(\boldsymbol{x}) f_{x_{1}}(\boldsymbol{x}) & f_{x_{2}}^{2}(\boldsymbol{x})
\end{array}\right) \mathrm{d} x_{1} \mathrm{~d} x_{2} \\
& =\left(\begin{array}{cc}
\left(w * f_{x_{1}}^{2}\right)\left(\boldsymbol{x}_{0}\right) & \left(w * f_{x_{1}} f_{x_{2}}\right)\left(\boldsymbol{x}_{0}\right) \\
\left(w * f_{x_{2}} f_{x_{1}}\right)\left(\boldsymbol{x}_{0}\right) & \left(w * f_{x_{2}}^{2}\right)\left(\boldsymbol{x}_{0}\right)
\end{array}\right),
\end{aligned}
$$

where $w * f$ denotes the convolution of $w$ and $f$. The partial derivative of $f$ with respect to some variable $x_{i}$ is denoted by $f_{x_{i}}$. This reveals that $\boldsymbol{J}$ is a smoothed version of

$$
\left(\begin{array}{cc}
f_{x_{1}}^{2}(\boldsymbol{x}) & f_{x_{1}}(\boldsymbol{x}) f_{x_{2}}(\boldsymbol{x}) \\
f_{x_{2}}(\boldsymbol{x}) f_{x_{1}}(\boldsymbol{x}) & f_{x_{2}}^{2}(\boldsymbol{x})
\end{array}\right) .
$$

The eigenvalues of the structure tensor are noted $\lambda_{\max }$ and $\lambda_{\min }$, with $\lambda_{\min }, \lambda_{\max } \in \mathbb{R}$. They carry information about the distribution of the gradient within the window $w$. Depending on the eigenvalues, one can discriminate between homogenous regions, rotational symmetric regions without predominant direction, regions where the eigenvector is well-aligned with one of the gradient directions, or regions where the dominant direction lies in between the gradient directions. For such purpose, two measures are defined, the so called energy $E$ and the coherence $C$. The energy is defined based on the eigenvalues of the structure tensor as $E=\left|\lambda_{1}\right|+\left|\lambda_{2}\right|$. If $E \approx 0$, which corresponds to $\lambda_{\max }=\lambda_{\min } \approx 0$, then the region is homogenous. If $E \gg 0$, then the characteristic of the structure is determined by the coherency information. The coherency information $C$ is a measure of confidence, defined as

$$
0 \leq C=\frac{\lambda_{\max }-\lambda_{\min }}{\lambda_{\max }+\lambda_{\min }}=\frac{\sqrt{\left(J_{22}-J_{11}\right)^{2}+4 J_{12}^{2}}}{J_{22}+J_{11}} \leq 1,
$$

where $J_{\mathrm{ij}}$ denotes an element of the structure tensor. If $C \approx 0$, which corresponds to $\lambda_{\text {max }} \approx \lambda_{\text {min }}$, then the region is rotational symmetric without predominant direction, the structure has no orientation. If $C \approx 1$, which corresponds to $\lambda_{\max }>0, \lambda_{\min } \approx 0$ or $\lambda_{\max } \gg \lambda_{\min }$, the eigenvector is well-aligned with one of the gradient directions. For $0<C<1$, the predominant orientation lies between the gradient directions. In general, a coherency close to 1 indicates that the structure in the image is locally $1 \mathrm{D}$, a coherency close to 0 indicates that there is no preferred direction.

The energy of the derivative in the direction $\boldsymbol{u}$ can be expressed as

$$
\left\|\mathrm{D}_{\boldsymbol{u}} f\right\|_{w}^{2}=\left\langle\boldsymbol{u}^{T} \nabla f, \boldsymbol{u}^{T} \nabla f\right\rangle_{w}=\boldsymbol{u}^{T}\langle\boldsymbol{\nabla} f, \boldsymbol{\nabla} f\rangle_{w} \boldsymbol{u}=\boldsymbol{u}^{T} \mathbf{J} \boldsymbol{u} .
$$

This means that, in the window centered around $\boldsymbol{x}_{0}$, the dominant orientation of the neighborhood can be computed by

$$
\boldsymbol{u}_{1}=\arg \max _{\|\boldsymbol{u}\|=1}\left\|\mathrm{D}_{\boldsymbol{u}} f\right\|_{w}^{2} .
$$

We interpret $\left\|\mathrm{D}_{\boldsymbol{u}} f\right\|_{w}^{2}$ as the average energy in the window defined by $w$ and centered at $\boldsymbol{x}_{0}$. Moreover, $\mathrm{D}_{\boldsymbol{u}} f=\langle\boldsymbol{\nabla} f, \boldsymbol{u}\rangle$ is the derivative in the direction of $\boldsymbol{u}$. The maximizing argument corresponds to the eigenvector with the largest eigenvalue of the structure tensor at $\boldsymbol{x}_{0}$. The dominant orientation of the pattern in the local window $w$ is computed as $\boldsymbol{u}_{1}=(\cos \theta, \sin \theta)$, with $\theta=\frac{1}{2} \arctan \left(\frac{2 J_{12}}{J_{22}-J_{11}}\right)$.

Figure 2 illustrates the improved robustness of the structure tensor in terms of the estimation of the orientation. Figure 3 provides another concrete example on the structuretensor analysis produced by the freely available OrientationJ plugin for Fiji/ImageJ. ${ }^{1}$ We

\footnotetext{
${ }^{1}$ Software available at http://bigwww.epfl.ch/demo/orientation/
} 

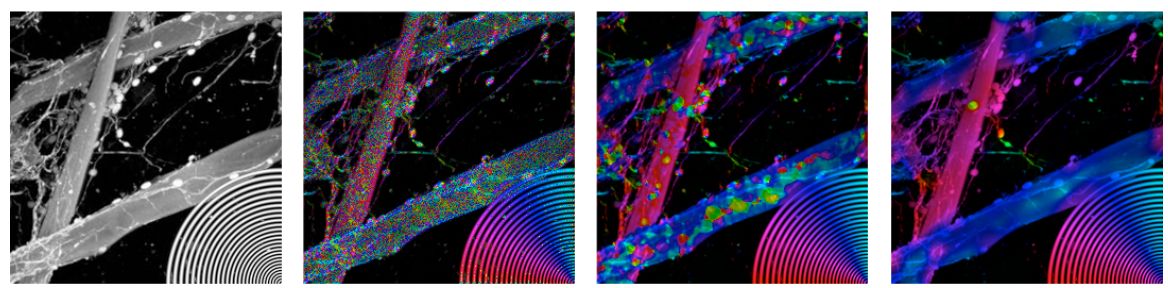

Fig. 2. Illustration of the robustness of the structure tensor in terms of estimation of the orientation, from left to right: (1) Input image: confocal micrograph, same as the original image in Figure 1. (2) Local dominant orientation, color-coded, no filtering applied. (3) Orientation given by the structure tensor with a small window size (standard deviation of the Gaussian window =1). (4) Orientation given by the structure tensor large window size (standard deviation of the Gaussian window $=1$ ). All the images were produced by the ImageJ/Fiji plugin OrientationJ.

have chosen a HSB (hue, saturation, and brightness) cylindrical-coordinate color representation to visualize the results. The HSB components correspond to the following values: angle of the orientation, coherency, and input image, respectively. The advantage of the proposed model is that it gives a direct link between the quantities to display and the color coding. In the cylindrical-coordinate color representation, the angle around the central vertical axis corresponds to hue. The distance along the axis corresponds to brightness, thus we preserve the visibility of the original structures. The distance from the axis corresponds to saturation: the higher the coherency is, the more saturated the corresponding colors are.

In the 3D shape estimation of DNA molecules from stereo cryo-electron micrographs (Fonck et al, 2008), the authors took advantage of its structure-tensor method. Other applications can be found in (Köthe, 2003) and (Bigun et al, 2004).

While simple and computationally efficient, the structure-tensor method has drawbacks: it only takes into account one specific scale, the localization accuracy for corners is low, and the integration of edge and corner detection is ad hoc (e.g. Harris' corner detector).

\subsection{Higher-Order Directional Structures and the Hessian}

To capture higher-order directional structures, the gradient information is replaced by higher-order derivatives. In general, an $n$ th-order directional derivative is associated with $n$ directions. Taking all of these to be the same, the directional derivative of order $n$ in $\mathbb{R}^{2}$ is defined as

$$
\mathrm{D}_{\boldsymbol{u}}^{n} f(\boldsymbol{x})=\sum_{k=0}^{n}\left(\begin{array}{l}
n \\
k
\end{array}\right) u_{1}^{k} u_{2}^{n-k} \partial_{x_{1}}^{k} \partial_{x_{2}}^{n-k} f(\boldsymbol{x}),
$$

which is a linear combination of partial derivatives of order $n$. More specifically, if we fix $n=2$ and the unit vector $\boldsymbol{u}_{\theta}=(\cos \theta, \sin \theta)$, we obtain

$$
\mathrm{D}_{\boldsymbol{u}_{\theta}}^{2} f(\boldsymbol{x})=\cos ^{2}(\theta) \partial_{x_{1}}^{2} f(\boldsymbol{x})+2 \cos (\theta) \sin (\theta) \partial_{x_{1}} \partial_{x_{2}} f(\boldsymbol{x})+\sin ^{2}(\theta) \partial_{x_{2}}^{2} f(\boldsymbol{x}) .
$$



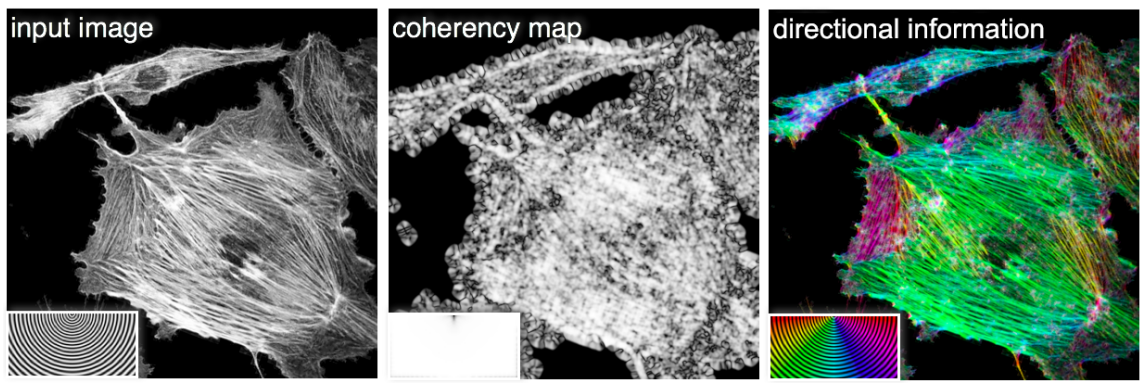

Fig. 3. Illustration of the use of structure tensors. Large images, from left to right: (1) Input image ( $800 \times 800$ pixels): immunofluorescence cytoskeleton (actin fibers), courtesy of Caroline Aemisegger, University of Zürich. (2) Coherency map: coherency values close to 1.0 are represented in white, coherency values close to 0.0 are represented in black. (3) Construction of color representation in HSB, H: angle of the orientation, S: coherency, B: input image. Small images in the left bottom corners, from left to right: (1) Input image: wave pattern with constant wavelength. (2) Coherency map: coherency values are close to 1.0 as expected. (3) The color representation reflects the different orientations. All the images were produced by the ImageJ/Fiji plugin OrientationJ.

The Hessian filter is a square matrix of second-order partial derivatives of a function. For example, in 2D, the smoothed Hessian matrix, useful for ridge detection at location $\boldsymbol{x}_{0}$, can be written as

$$
\mathbf{H}\left(x_{0}\right)=\left(\begin{array}{ll}
\left(w_{11} * f\right)\left(\boldsymbol{x}_{0}\right) & \left(w_{12} * f\right)\left(\boldsymbol{x}_{0}\right) \\
\left(w_{21} * f\right)\left(\boldsymbol{x}_{0}\right) & \left(w_{22} * f\right)\left(\boldsymbol{x}_{0}\right)
\end{array}\right),
$$

where $w$ is a smoothing kernel and $w_{\mathrm{ij}}=\partial_{x_{\mathrm{i}}} \partial_{x_{\mathrm{j}}} w$ denotes its derivatives with respect to the coordinates $x_{\mathrm{i}}$ and $x_{\mathrm{j}}$. In the window centered around $x_{0}$, the dominant orientation of the ridge is

$$
\boldsymbol{u}_{2}=\arg \max _{\|\boldsymbol{u}\|=1}\left(\boldsymbol{u}^{T} \mathbf{H} \boldsymbol{u}\right)
$$

The maximizing argument corresponds to the eigenvector with the largest eigenvalue of the Hessian at $\boldsymbol{x}_{0}$. The eigenvectors of the Hessian are orthogonal to each other, so the eigenvector with the smallest eigenvalue corresponds to the direction orthogonal to the ridge.

A sample application of the Hessian filter is vessel enhancement (Frangi et al, 1998). There, the authors define a measure called vesselness which corresponds to the likeliness of an image region to contain vessels or other image ridges. The vesselness measure is derived based on the eigenvalues of the steerable Hessian filter. In 2D, a vessel is detected when one of the eigenvalues is close to zero $\left(\lambda_{1} \approx 0\right)$ and the other one is much larger $\left|\lambda_{2}\right| \gg\left|\lambda_{1}\right|$. The direction of the ridge is given by the eigenvector of the Hessian filter output corresponding to $\lambda_{1}$. In (Frangi et al, 1998), Frangi et al. defines the measure of vesselness as

$$
V(\boldsymbol{x})= \begin{cases}0, & \text { if } \lambda_{1}>0 \\ \exp \left(-\frac{\left(\lambda_{1} / \lambda_{2}\right)^{2}}{2 \beta_{1}}\right)\left(1-\exp \left(-\frac{\lambda_{1}^{2}+\lambda_{2}^{2}}{2 \beta_{2}}\right)\right), & \text { otherwise }\end{cases}
$$



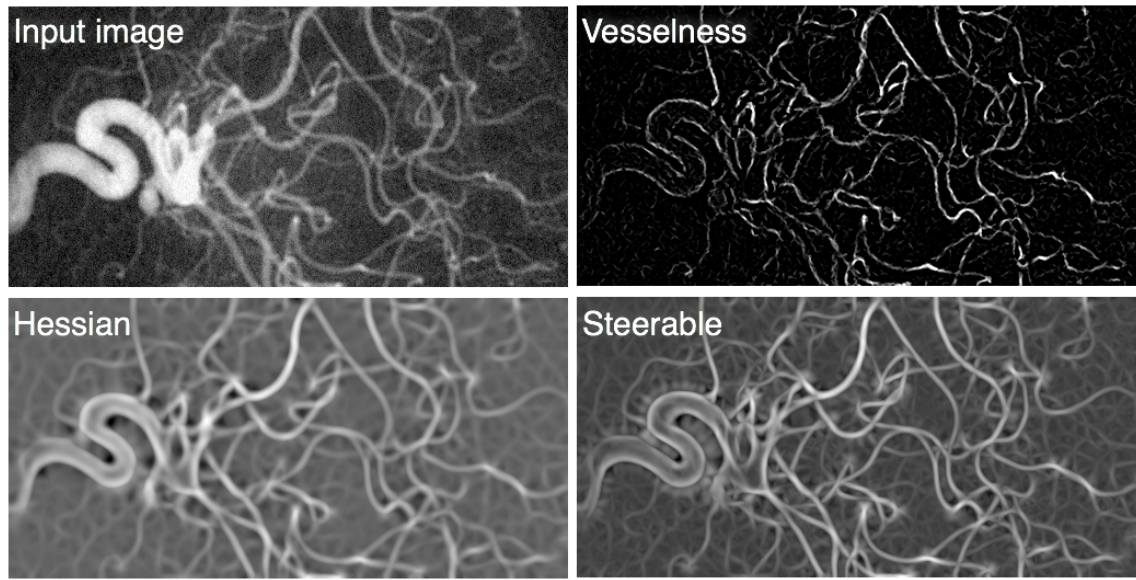

Fig. 4. Rotation-invariant enhancement of filaments. From top to bottom, left to right: (1) Input image (512 x 256 pixels) with neuron, cell body, and dendrites (maximum-intensity projection of a z-stack, fluorescence microscopy, inverted scale). (2) Output of the Hessian filter. The largest eigenvalue of the Hessian matrix was obtained after a Gaussian smoothing (standard deviation $=5$ ). The image was produced using the ImageJ/Fiji plugin FeatureJ available at: http://www.imagescience.org/meijering/software/featurej/. (3) Output of the vesselness index obtained by the Fiji plugin Frangi-Vesselness. (4) Output of the steerable filters (Gaussian-based, 4th order). The image was produced using the ImageJ/Fiji plugin SteerableJ.
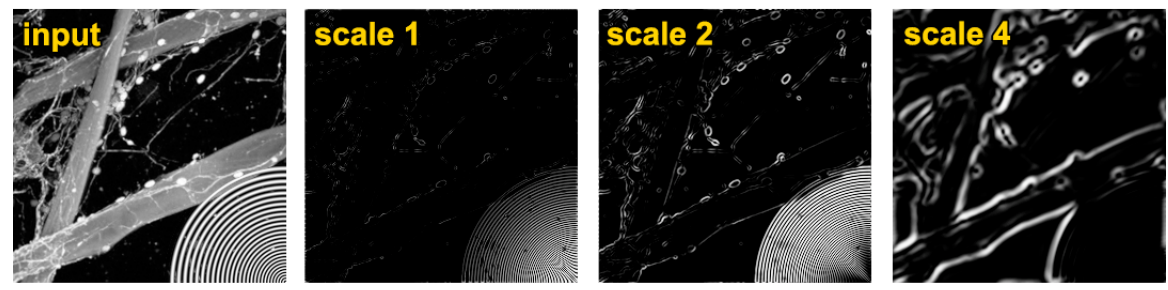

Fig. 5. Illustration of the gradient at different scales, from left to right: (1) Input image: confocal micrograph, same as the original image in Figure 1. (2) Magnitude of the gradient at scale 1. (3) Magnitude of the gradient at scale 2. (4) Magnitude of the gradient at scale 4. All the images were produced by the ImageJ/Fiji plugin OrientationJ.

where $\beta_{1}$ and $\beta_{2}$ control the sensitivity of the filter ${ }^{2}$. A particular application of the vesselness index on filament enhancement is shown in Figure 4. Alternative vesselness measures based on the Hessian have been proposed by Lorenz et al. (Lorenz et al, 1997) and Sato et al. (Sato et al, 1998).

\footnotetext{
${ }^{2}$ Plugin available at http://fiji.sc/Frangi/
} 


\section{Directional Multiscale Approaches}

In natural images, oriented patterns are typically living on different scales, for example, thin and thick blood vessels. To analyze them, methods that extract oriented structures separately at different scales are required. The classical tools for a multiscale analysis are wavelets. In a nutshell, a wavelet is a bandpass filter that responds almost exclusively to features of a certain scale. The separable wavelet transform that is commonly used is computationally very efficient but provides only limited directional information. Its operation consists of filtering with 1D wavelets with respect to the horizontal and vertical directions. As a result, two pure orientations (vertical and horizontal), and a mixed channel of diagonal directions are extracted. Using the dual-tree complex wavelet transform (Kingsbury, $1998)^{3}$, one can increase the number of directions to six while retaining the computational efficiency of the separable wavelet transform. (We refer to (Selesnick et al, 2005) for a detailed treatment of this transform.) Next, we describe how to achieve wavelets with an even higher orientational selectivity at the price of higher computational costs.

Figure 5 illustrates the gradient at different scales. We can observe that different features are kept at different scales.

\subsection{Construction of Directional Filters in the Fourier Domain}

In order to construct orientation-selective filters, methods based on the Fourier transform are powerful. From now on, we denote the Cartesian and polar representations of the same 2D function $f$ by $f(\boldsymbol{x})$ with $\boldsymbol{x} \in \mathbb{R}^{2}$ and $f_{\text {pol }}(r, \theta)$ with $r \in \mathbb{R}^{+}, \theta \in[0,2 \pi)$ (similarly in the Fourier domain: $\hat{f}(\boldsymbol{\omega})$ and $\left.\hat{f}_{\mathrm{pol}}(\rho, \phi)\right)$. The key property for directional analysis is that rotations in the spatial domain propagate as rotations to the Fourier domain. Formally, we write that

$$
f\left(\mathbf{R}_{\theta} \boldsymbol{x}\right) \stackrel{\mathscr{F}}{\longleftrightarrow} \hat{f}\left(\mathbf{R}_{\theta} \boldsymbol{\omega}\right),
$$

where $\mathbf{R}_{\theta}$ denotes a rotation by the angle $\theta$. The construction is based on a filter $\psi$ whose Fourier transform $\widehat{\psi}$ is supported on a wedge around the $\omega_{1}$ axis; see Figure 6 . In order to avoid favoring special orientations, one typically requires that $\hat{\psi}$ be nonnegative and that it forms (at least approximately) a partition of unity of the Fourier plane under rotation, like

$$
\sum_{\theta_{i}}\left|\widehat{\psi}\left(\mathbf{R}_{\theta_{i}} \boldsymbol{\omega}\right)\right|^{2}=1 \text {, for all } \boldsymbol{\omega} \in \mathbb{R}^{2} \backslash\{\mathbf{0}\} .
$$

Here, $\theta_{1}, \ldots, \theta_{n}$ are arbitrary orientations which are typically selected to be equidistant, with $\theta_{i}=(i-1) \pi / n$. To get filters that are well localized in the spatial domain, one chooses $\hat{\psi}$ to be a smooth function; for example the Meyer window function (Daubechies, 1992; Ma and Plonka, 2010). A directionally filtered image $f_{\theta_{i}}$ can be easily computed by rotating the window $\hat{\psi}$ by $\theta_{i}$ and multiplying it with the Fourier transform $\hat{f}$ of the image, and by transforming back to the spatial domain. This is written

$$
f_{\theta_{i}}(\boldsymbol{x})=\mathscr{F}^{-1}\left\{\hat{\psi}\left(\mathbf{R}_{\theta_{i}} \cdot\right) \hat{f}\right\}(\boldsymbol{x}) .
$$

(We refer to (Chaudhury et al, 2010) for filterings based on convolutions in the spatial domain.) The resulting image $f_{\theta_{i}}$ contains structures that are oriented along the direction $\theta_{i}$. The local orientation $\theta$ is given by the orientation of the maximum filter response

\footnotetext{
${ }^{3}$ Available at http://eeweb.poly.edu/iselesni/WaveletSoftware/
} 

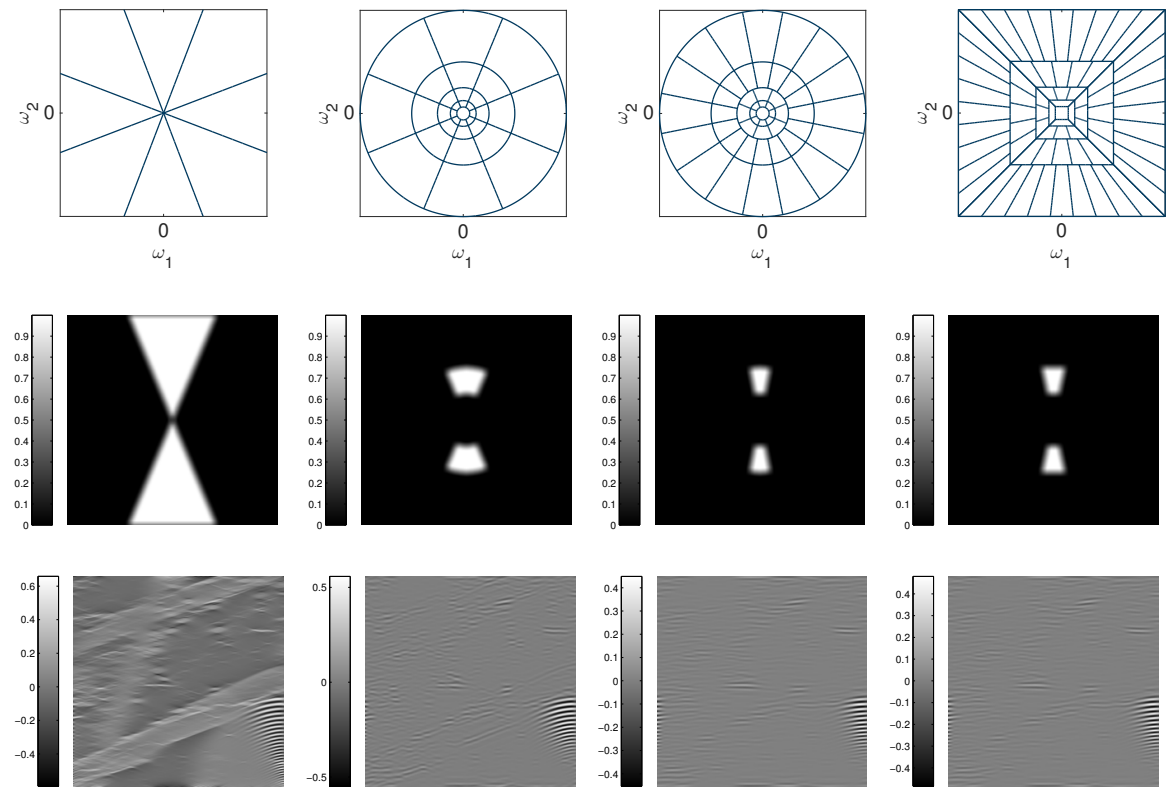

Fig. 6. Top row (from left to right): schematic tilings of the frequency plane by Fourier filters, directional wavelets, curvelets, and shearlets (the origin of the Fourier domain lies in the center of the images); Middle row: a representative Fourier multiplier. Bottom row: Corresponding filtering result for the image of Figure 1. The Fourier filter extracts oriented patterns at all scales whereas the wavelet-type approaches are sensitive to oriented patterns of a specific scale. Curvelets and shearlets additionally increase the directional selectivity at the finer scales.

$$
\theta(\boldsymbol{x})=\arg \max _{\theta_{i}}\left|f_{\theta_{i}}(\boldsymbol{x})\right| .
$$

Such directional filters have been used in fingerprint enhancement (Sherlock et al, 1994) and in crossing-preserving smoothing of images (Franken and Duits, 2009; Franken et al, 2007).

\subsection{Directional Wavelets with a Fixed Number of Directions}

Now we augment the directional filters by scale-selectivity. Our starting point is the radial windowing function of (15). The simplest way to construct a directional wavelet transform is to partition the Fourier domain into dyadic frequency bands ("octaves"). To ensure a complete covering of the frequency plane, we postulate again nonnegativity and a partition-of-unity property of the form

$$
\sum_{s \in \mathbb{Z}} \sum_{\theta_{i}}\left|\widehat{\psi}\left(2^{-s} \mathbf{R}_{\theta_{i}} \boldsymbol{\omega}\right)\right|^{2}=1 \text {, for } \boldsymbol{\omega} \in \mathbb{R}^{2} \backslash\{\mathbf{0}\} .
$$

Classical examples of this type are the Gabor wavelets that cover the frequency plane using Gaussian windows which approximate (rescaled) partition-of-unity (Lee, 1996; Mal- 
lat, 2008). These serve as model for the filters in the mammalian visual system (Daugman, 1985, 1988). Alternative constructions are Cauchy wavelets (Antoine et al, 1999) or constructions based on the Meyer window functions (Daubechies, 1992; Ma and Plonka, 2010). We refer to (Vandergheynst and Gobbers, 2002) and (Jacques et al, 2011) for further information on the design of directional wavelets. In particular, sharply direction-selective Cauchy wavelets have been used for symmetry detection (Antoine et al, 1999).

\subsection{Curvelets, Shearlets, Contourlets, and Related Transforms}

Over the past decade, curvelets (Candès and Donoho, 2004), shearlets (Kutyniok and Labate, 2012; Labate et al, 2005; Yi et al, 2009), and contourlets (Do and Vetterli, 2005) have attracted a lot of interest. They are constructed similarly to the directional wavelets. The relevant difference in this context is that they increase the directional selectivity on the finer scales according to a parabolic scaling law. This means that the number of orientations is increased by a factor of about $\sqrt{2}$ at every scale or by 2 at every other scale; see Figure 6. Therefore, they are collectively called parabolic molecules (Grohs and Kutyniok, 2014). Curvelets are created by using a set of basis functions from a series of rotated and dilated versions of an anisotropic mother wavelet to approximate rotation and dilation invariance. Contourlets use a tree-structured filterbank to reproduce the same frequency partitioning as curvelets. Their structure is more flexible, enabling different subsampling rates. To overcome the limitations of the Cartesian grid (i.e., exact rotation invariance is not achievable on it), shearlets are designed in the discrete Fourier domain with constraints on exact sheer invariance.

These transforms are well suited to the analysis and synthesis of images with highly directional features. Applications include texture classification of tissues in computed tomography (Semler and Dettori, 2006), texture analysis (Dong et al, 2015), image denoising (Starck et al, 2002), contrast enhancement (Starck et al, 2003), and reconstruction in limited-angle tomography (Frikel, 2013). Furthermore, they are closely related to a mathematically rigorous notion of the orientation of image features, the so-called wavefront set (Candès and Donoho, 2005; Kutyniok and Labate, 2009). Loosely speaking, the wavefront set is the collection of all edges along with their normal directions. This property is used for the geometric separation of points from curvilinear structures, for instance, to separate spines and dendrites (Kutyniok, 2012) and for edge detection with resolution of overlaying edges (Guo et al, 2009; Storath, 2011b; Yi et al, 2009). We show in Figure 7 the result of the curvelet/shearlet-based edge-detection scheme of (Storath, 2011b) which is obtained as follows: For every location (pixel) $\boldsymbol{b}$ and every available orientation $\theta$, the rate of decay $d_{\boldsymbol{b}, \theta}$ of the absolute values of the curvelet/shearlet coefficients over the scale is computed. The reason for computing the rate of decay of the coefficients is their connection to the local regularity: the faster the decay rate, the smoother the image at location $\boldsymbol{b}$ and orientation $\theta$ (see (Candès and Donoho, 2005; Guo et al, 2009; Kutyniok and Labate, 2009)). We denote the curvelet/shearlet coefficients at scale $a$, location $\boldsymbol{b}$, and orientation $\theta$ by $c_{a, \boldsymbol{b}, \theta}$. Then,

$d_{\boldsymbol{b}, \theta}$ corresponds to the least-squares fit to the set of constraints $\left|c_{a, \boldsymbol{b}, \theta}\right|=C_{\boldsymbol{b}, \theta}^{\prime} a^{d_{\boldsymbol{b}, \theta}}$, where $a$ runs over all available scales (in the example of Figure $7, a=2^{-s / 3}$ with $s=0, \ldots, 15$ ). Note that this reduces to solving a system of linear equations in terms of $\log C_{\boldsymbol{b}, \theta}^{\prime}$ and $d_{\boldsymbol{b}, \theta}$, after having taken a logarithm on both sides. Having computed $d$, we perform for each orientation $\theta$ a non-maximum suppression on $d$; that is, we set to $(-\infty)$ all pixels that are not a local maximum of the image $d_{,, \theta}$ with respect to the direction $\theta$. Finally, a threshold is applied and the connected components of the (3D-array) $d$ are determined 

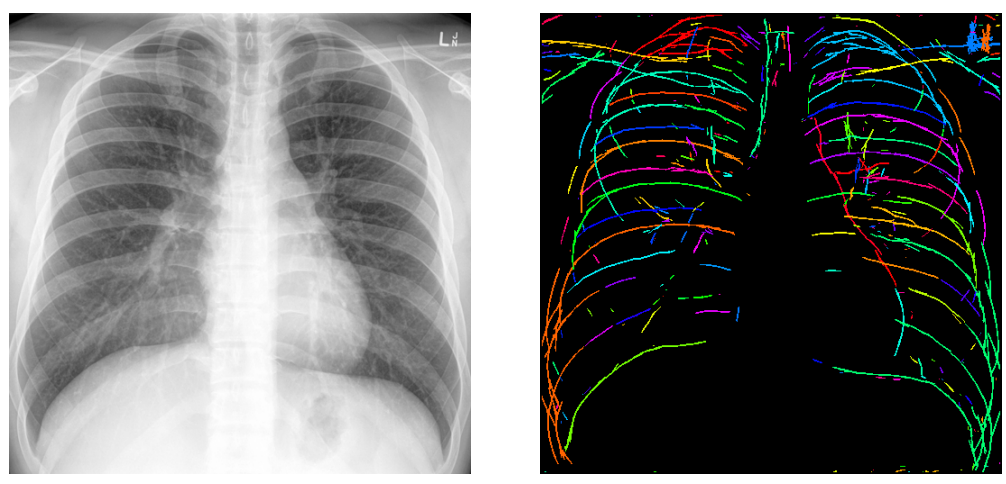

Fig. 7. Edge detection with resolution of crossing edges using the curvelet transform. The colors correspond to connected edge segments. Note that crossing edges are resolved, for instance near the shoulder bones. [Original image courtesy of Dr. Jeremy Jones, Radiopaedia.org]

(and colored). The image displayed in Figure 7 is the maximum-intensity projection of the three-dimensional image $d$ with respect to the $\theta$ component.

Relevant software packages implementing these transforms are the Matlab toolboxes CurveLab $^{4}$, ShearLab ${ }^{5}$, FFST $^{6}$, and the 2D Shearlet Toolbox ${ }^{7}$.

\section{Steerable Filters}

For the purpose of detecting or enhancing a given type of directional pattern (edge, line, ridge, corner), a natural inclination is to try to match directional patterns. The simplest way to do that is to construct a template and try to align it with the pattern of interest. Usually, such algorithms rely on the discretization of the orientation. To obtain accurate results, a fine discretization is required. In general, Fourier filters and wavelet transforms are computationally expensive in this role because a full $2 \mathrm{D}$ filter operation has to be computed for each discretized direction. However, an important exception is provided by steerable filters, where one may perform arbitrary (continuous) rotations and optimizations with a substantially reduced computational overhead. The basics of steerability were formulated by Freeman and Adelson in the early nineties (Freeman and Adelson, 1990, 1991; Freeman, 1992) and developed further by Perona (Perona, 1992), Simoncelli and Farid (Simoncelli and Farid, 1996), and Unser et al. (Unser and Chenouard, 2013; Unser and Van De Ville, 2010; Ward and Unser, 2014; Ward et al, 2013). We now explain the property of steerability and show the development of steerable wavelets.

A function $f$ on the plane is steerable in the finite basis $\left\{f_{1}, \ldots, f_{N}\right\}$ if, for any rotation matrix $\mathbf{R}_{\theta_{0}}$, we can find coefficients $c_{1}\left(\theta_{0}\right), \ldots, c_{N}\left(\theta_{0}\right)$ such that

\footnotetext{
${ }^{4}$ Available at http://www.curvelet.org/

${ }^{5}$ Available at http://www.shearlab.org/

${ }^{6}$ Available at http://www.mathematik.uni-kl.de/imagepro/members/haeuser/ffst/

${ }^{7}$ Available at http://www.math.uh.edu/ dlabate/software.html
} 


$$
f\left(\mathbf{R}_{\theta_{0}} \boldsymbol{x}\right)=\sum_{n=1}^{N} c_{n}\left(\theta_{0}\right) f_{n}(\boldsymbol{x}) .
$$

It means that a function $f$ in $\mathbb{R}^{2}$ is steerable if all of its rotations can be expressed in the same finite basis as the function itself. Thus, any rotation of $f$ can be computed with a systematic modification (i.e., matrix multiplication) of the coefficients. The importance of this property is that, when doing pattern matching, it is enough to compute the coefficients only once, for one particular angle. Based on that, one can then easily determine the coefficients for arbitrary angles. A simple example of steerable functions are the polarseparable functions, whose amplitude (radial part) is 1 and angular part is $\cos (\theta)$ or $\sin (\theta)$. All rotations of these functions can be expressed in the basis $\{\sin (\theta), \cos (\theta)\}$. The rotations of $\cos (\theta)$ and $\sin (\theta)$ by $\theta_{0}$ are determined by

$$
\left(\begin{array}{lr}
\cos \left(\theta+\theta_{0}\right) \\
\sin \left(\theta+\theta_{0}\right)
\end{array}\right)=\left(\begin{array}{rr}
\cos \left(\theta_{0}\right) & -\sin \left(\theta_{0}\right) \\
\sin \left(\theta_{0}\right) & \cos \left(\theta_{0}\right)
\end{array}\right)\left(\begin{array}{l}
\cos (\theta) \\
\sin (\theta)
\end{array}\right) .
$$

Instead of setting the amplitude to 1 , one can choose any nonvanishing isotropic function for the radial part. Also, replacing sin and cos with exponentials will preserve the property (since $\mathrm{e}^{\mathrm{j}\left(\theta+\theta_{0}\right)}=\mathrm{e}^{\mathrm{j} \theta_{0}} \mathrm{e}^{\mathrm{j} \theta}$ ).

The simplest examples of steerable filters are the ones that are based on the gradient or the Hessian. Starting from an isotropic lowpass function $\varphi\left(x_{1}, x_{2}\right)$, one can create a subspace of steerable derivative-based templates which can serve as basic edge or ridge detectors. In 2D, let $\varphi_{k, l}=\partial_{x_{1}}^{k} \partial_{x_{2}}^{l} \varphi$ be anisotropic derivatives of the isotropic function $\varphi$. By the chain rule of differentiation, for any rotation matrix $\mathbf{R}_{\theta_{0}}$, the function $\partial_{x_{1}}^{k} \partial_{x_{2}}^{l} \varphi\left(\mathbf{R}_{\theta_{0}}\right.$ ) can be written as a linear combination of $\varphi_{i, j}$ with $i+j=k+l$. Therefore, any anisotropic filter of the form

$$
h\left(x_{1}, x_{2}\right)=\sum_{m=1}^{M} \sum_{k+l=m} \alpha_{k, l} \varphi_{k, l}\left(x_{1}, x_{2}\right)
$$

is steerable. Consequently, for any rotation matrix $\mathbf{R}_{\theta_{0}}$, an application of the rotated filter to an image $f$ yields

$$
\left(f * h\left(\mathbf{R}_{\theta} \cdot\right)\right)(\boldsymbol{x})=\sum_{m=1}^{M} \sum_{k+l=m} \alpha_{k, l}(\theta) f_{k, l}(\boldsymbol{x}),
$$

where $f_{k, l}=f * \partial_{x_{1}}^{k} \partial_{x_{2}}^{l} \varphi$ and $\alpha_{k, l}(\theta)$ is a trigonometric polynomial in $\cos (\theta)$ and $\sin (\theta)$. Once every $f_{k, l}$ is precomputed, the linear combination (22) allows us to quickly evaluate the filtering of the image by the anisotropic filter rotated by any angle. We can then „steer” $h$ by manipulating $\theta$, typically to determine the direction along which the response is maximized and across which is minimized.

Figure 4 contains the outcome of three derivative-based methods used to enhance filaments in a rotation-invariant way. The computation complexity is the same for the different methods, with approximatively the same number of filters with the same computation time. The directionality is best captured in the steerable case.

Jacob and Unser (Jacob and Unser, 2004) improved the basic steerable filters by imposing Canny-like criteria of optimality (Canny, 1986) on the properties of the detector: reasonable approximation of the ideal detector, maximum signal-to-noise ratio, good spatial localization, and reduced oscillations. Their formalism boils down to a constrained optimization of the expansion coefficients $\alpha_{k, i}$ using Lagrange multipliers. 


\section{Steerable Multiscale Approaches}

In (Simoncelli and W.T., 1995), Simoncelli et al. proposed a new take on steerable filters: the steerable pyramid. The goal of his design was to combine steerability with a multiscale detection scheme. His pioneering work had many successful applications: contour detection (Perona, 1992), image filtering and denoising (Bharath and Ng, 2005), orientation analysis (Simoncelli and Farid, 1996), and texture analysis and synthesis (Portilla and Simoncelli, 2000). In (Karssemeijer and te Brake, 1996) multiscale steerable filters were involved in the detection of stellate distortions in mammograms. Classical multiresolution steerable methods use a purely discrete framework with no functional analytic counterpart. They are not amenable to extensions to dimensions higher than two. Fortunately, it is possible to address these limitations. In this section we overview a continuous-domain formulation that extends the technique proposed by Simoncelli et al. in (Simoncelli and W.T., 1995). Multiresolution directional techniques were motivated by their invariance with respect to primary geometric transformations: translation, dilation, and rotation. Translation and dilation invariance is satisfied by the application of the wavelet transform. Rotation invariance is achieved by the Riesz transform, which also gives a connection to gradient-like signal analysis (Held et al, 2010).

\subsection{The Riesz Transform}

The complex Riesz transform was introduced to the literature by Larkin (Larkin, 2001; Larkin et al, 2001) as a multidimensional extension of the Hilbert transform. The Hilbert transform is a 1D shift-invariant operator that maps all cosine functions into sine functions without affecting their amplitude (allpass filter). Expressed in the Fourier domain, the Hilbert transform of a function $f$ is

$$
\mathscr{F}\{\mathscr{H}\{f\}\}(\omega)=-\frac{\mathrm{j} \omega}{|\omega|} \hat{f}(\omega)=-\mathrm{j} \operatorname{sgn}(\omega) \hat{f}(\omega) .
$$

Similarly to the Hilbert transform, the Riesz transform is defined in the Fourier domain as

$$
\mathscr{F}\{\mathscr{R}\{f\}\}(\boldsymbol{\omega})=\frac{\left(\omega_{x}+\mathrm{j} \omega_{y}\right)}{\|\boldsymbol{\omega}\|} \hat{f}(\boldsymbol{\omega})=\mathrm{e}^{\mathrm{j} \phi} \hat{f}_{\text {pol }}(\rho, \phi),
$$

where the subscript "pol" denotes the polar representation of the function. The transform is a convolution-type operator that also acts as an allpass filter, with a phase response that is completely encoded in the orientation.

The Riesz transform is translation- and scale-invariant since

$$
\begin{gathered}
\forall x_{0} \in \mathbb{R}^{d}, \quad \mathscr{R}\left\{f\left(\cdot-\boldsymbol{x}_{0}\right)\right\}(\boldsymbol{x})=\mathscr{R} f\left(\boldsymbol{x}-\boldsymbol{x}_{0}\right) \\
\forall a \in \mathbb{R}^{+} \backslash\{0\}, \quad \mathscr{R}\left\{f\left(\frac{\dot{a}}{a}\right)\right\}(\boldsymbol{x})=\mathscr{R} f\left(\frac{\boldsymbol{x}}{a}\right) .
\end{gathered}
$$

The Riesz transform is also rotation-invariant.

The $n$ th-order complex 2D Riesz transform $\mathscr{R}^{n}$ represents the $n$-fold iterate of $\mathscr{R}$, defined in the Fourier domain as

$$
\mathscr{F}\left\{\mathscr{R}^{n}\{f\}\right\}(\rho \cos \phi, \rho \sin \phi)=\mathrm{e}^{\mathrm{j} n \phi} \hat{f}_{\text {pol }}(\rho, \phi) .
$$

The $n$-th order Riesz transform decomposes a $2 \mathrm{D}$ signal into $n+1$ distinct components. It inherits the invariance properties of the Riesz transform since they are preserved through iteration. This means that we can use the Riesz transform to map a set of primary wavelets into an augmented one while preserving the scale- and shift-invariant structure. 


\subsection{Connection to the Gradient and Directional Derivatives}

In this section, we describe the connection between the Riesz transform, the directional Hilbert transform, the gradient, and the directional derivatives. Assuming a zero-mean function $f$, the Riesz transform is related to the complex gradient operator as

$$
\mathscr{R} f\left(x_{1}, x_{2}\right)=-\mathrm{j}\left(\frac{\partial}{\partial x_{1}}+\mathrm{j} \frac{\partial}{\partial x_{2}}\right)(-\Delta)^{-1 / 2} f\left(x_{1}, x_{2}\right) .
$$

Here, $(-\Delta)^{\alpha}, \alpha \in \mathbb{R}^{+}$is the isotropic fractional differential operator of order $2 \alpha$. Conversely, the corresponding fractional integrator of order $2 \alpha$ is $(-\Delta)^{-\alpha}, \alpha \in \mathbb{R}^{+}$. The value $\alpha=1 / 2$ is of special interest, providing the link between the Riesz transform and the complex gradient operator. The integral operator acts on all derivative components and has an isotropic smoothing effect, thus, the Riesz transform acts as the smoothed version of the image gradient.

Assuming a zero-mean function $f$, the high-order Riesz transform is related to the partial derivatives of $f$ by

$$
\mathscr{R}^{n} f\left(x_{1}, x_{2}\right)=(-\Delta)^{-\frac{n}{2}} \sum_{n_{1}=0}^{n}\left(\begin{array}{c}
n \\
n_{1}
\end{array}\right)(-\mathrm{j})^{n_{1}} \partial_{x_{1}}^{n_{1}} \partial_{x_{2}}^{n-n_{1}} f\left(x_{1}, x_{2}\right) .
$$

The fractional integrator acts as an isotropic lowpass filter whose smoothing strength increases with $n$. The Riesz transform captures the same directional information as derivatives. However, it has the advantage of being better conditioned since, unlike them, it does not amplify the high frequencies.

The directional Hilbert transform is the Hilbert transform along a direction $\boldsymbol{u}$. It is related to the Riesz transform by

$$
\mathscr{H}_{\boldsymbol{u}_{\theta}} f(\boldsymbol{x})=\cos \theta f_{1}(\boldsymbol{x})+\sin \theta f_{2}(\boldsymbol{x}),
$$

where $f_{1}=\operatorname{Re}(\mathscr{R} f)$ and $f_{2}=\operatorname{Im}(\mathscr{R} f)$ are the real and imaginary parts of $\mathscr{R} f$. Assuming again a zero-mean function $f$, the directional Hilbert transform is related to the derivative in the direction $\boldsymbol{u}$ by

$$
\mathscr{H}_{\boldsymbol{u}} f(\boldsymbol{x})=-(-\Delta)^{-\frac{1}{2}} \mathrm{D}_{\boldsymbol{u}} f(\boldsymbol{x})
$$

Here, the operator $\mathrm{D}_{\boldsymbol{u}}$ is the one defined in (1). This result corresponds to the interpretation that the Hilbert transform acts as a lowpass-filtered version of the derivative operator. The $n$-fold version of the directional Hilbert transform acting on a zero-mean function $f$ along the direction specified by $\boldsymbol{u}$ can be expressed in term of the partial derivatives of $f$ as

$$
\mathscr{H}_{\boldsymbol{u}}^{n} f(\boldsymbol{x})=(-1)^{n}(-\Delta)^{-\frac{n}{2}} \mathrm{D}_{\boldsymbol{u}}^{n} f(\boldsymbol{x})
$$

\subsection{Steerable Wavelets}

In this section, we present the construction of steerable wavelet frames that are shaped to capture the local orientation of features in images within a multiresolution hierarchy. Their construction has two main parts: first, generation of circular harmonic wavelet frames by applying the multiorder complex Riesz transform on a bandlimited isotropic 

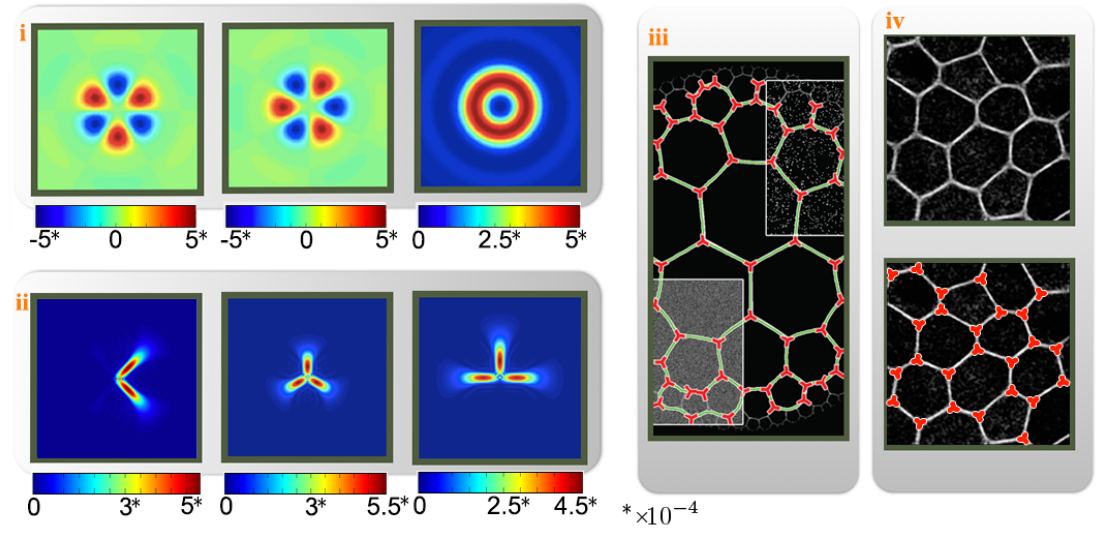

Fig. 8. From left to right: i., Circular harmonic wavelets of order three, real part, imaginary part, and magnitude; ii., steerable wavelets (different shapes, magnitude); iii., detections in synthetic data exhibiting multiple scales; iv., original image and detections in a micrograph of embryonic stem cells.

mother wavelet; second, shaping of the wavelet frames to a particular desired profile with an orthogonal transform.

We start our construction of steerable wavelet frames from a bandlimited isotropic mother wavelet in $L_{2}\left(\mathbb{R}^{2}\right)$, denoted by $\psi$, whose shifts and dilations form a wavelet frame (e.g., Simoncelli's wavelet). This isotropic wavelet at scale $s$ and grid point (location) $x_{0}=$ $2^{s} \boldsymbol{k}, \boldsymbol{k} \in \mathbb{Z}^{2}$ (in 2D), takes the form of

$$
\psi_{s}\left(\boldsymbol{x}-\boldsymbol{x}_{0}\right)=\frac{1}{2^{s}} \psi\left(\frac{\boldsymbol{x}-\boldsymbol{x}_{0}}{2^{s}}\right)=\frac{1}{2^{s}} \psi\left(\frac{\boldsymbol{x}}{2^{s}}-\boldsymbol{k}\right) .
$$

We then apply the multiorder complex Riesz transform on $\psi_{s}\left(\cdot-\boldsymbol{x}_{0}\right)$. The transform preserves the frame properties. Thus, by choosing $N$ distinct values for the integer $n$ (distinct set of harmonics), one can form a frame of steerable wavelets, referred to as circular harmonic wavelets (Jacovitti and Neri, 2000). An $n$ th-order harmonic wavelet has a rotational symmetry of order $n$ around its center, corresponding to the $n$ th-order rotational symmetry of $\mathrm{e}^{\mathrm{j} n \phi}$. The illustration of circular harmonics for order three is presented in Figure 8 (i.). An application of circular harmonic wavelets on local symmetry detection is presented in (Püspöki and Unser, 2015).

\subsection{Detection of Junctions}

An important step in the analysis of microscopic images is the detection of key points, or junctions of coinciding branches. The automatic detection of these junctions can facilitate further image-processing tasks such as cell segmentation, counting of cells, or image statistics. $M$-fold symmetric structures (including the case of ridges, assimilated to twofold symmetries) are present in filaments, fibers, membranes, or endothelial cells (e.g., in the eyes). The difficulty in the detection of these junctions is twofold. First, they can appear at arbitrary orientation and scale; second, biological micrographs are frequently contaminated by local variations in intensity and measurement noise. With the modified 
wavelet schemes presented above, one can design an efficient detector of the location and orientation of local symmetries.

From the circular harmonic wavelet representation, one constructs new steerable representations by using an orthogonal shaping matrix $\mathbf{U}$ to define new steerable-frame functions (Unser and Chenouard, 2013). The role of the shaping matrix $\mathbf{U}$ is to give the wavelet functions a desired angular profile. The transform can be formulated as

$$
\left[\begin{array}{c}
\xi_{s, \boldsymbol{k}}^{\left(n_{1}\right)} \\
\vdots \\
\xi_{s, \boldsymbol{k}}^{\left(n_{N}\right)}
\end{array}\right]=\mathbf{U}\left[\begin{array}{c}
\psi_{s, \boldsymbol{k}}^{(1)} \\
\vdots \\
\psi_{s, \boldsymbol{k}}^{(N)}
\end{array}\right]
$$

where $\psi_{s, \boldsymbol{k}}^{(n)}=\mathscr{R}^{n} \psi_{s, \boldsymbol{k}}$ is the $n$ th-order circular harmonic wavelet at scale $s$ and location $\boldsymbol{k}, \mathbf{U}$ is an orthonormal transformation matrix and $\left\{\xi_{s, \boldsymbol{k}}^{(n)}\right\}$ are the new wavelet channels at scale $s$ and location $\boldsymbol{k}$. The number of channels corresponds to the number of harmonics. The new wavelets span the same space as the wavelet frame $\psi_{s, \boldsymbol{k}}^{(n)}$.

Specific detectors are designed by defining the right weights for $\mathbf{U}$. Typically, the process involves an optimization problem, either in the space or in the Fourier domain. The list of coefficients to optimize can be expressed as a vector that takes the form $\boldsymbol{u}=\left(u_{1}, \ldots, u_{N}\right)$, with $\boldsymbol{u} \boldsymbol{u}^{\mathrm{H}}=1$. One can specify a quadratic energy term to minimize in the space domain like

$$
E[\xi]=\frac{1}{2 \pi} \int_{0}^{\infty} \int_{-\pi}^{\pi}|\xi(r, \theta)|^{2} \nu(\theta) r \mathrm{~d} \theta \mathrm{d} r
$$

or (for symmetric patterns) in the Fourier domain like

$$
E[\hat{\xi}]=\frac{1}{2 \pi} \int_{0}^{\infty} \int_{-\pi}^{+\pi}|\hat{\xi}(\rho, \phi)|^{2} v(\phi) \rho \mathrm{d} \phi \mathrm{d} \rho .
$$

The angular weighting function $v(\theta) \geq 0$ or $v(\phi) \geq 0$ should have minima on the unit circle that enforces the concentration of the energy along the desired pattern (for instance, symmetric shape or T-shape). Minimizing $E$ will thus force the solution $\xi$ to be localized at the corresponding angles. Once the mother wavelet $\xi$ is found, its translates and dilates naturally share the same optimal angular profile around their center. By expanding $\xi$ as $\sum_{n} u_{n} \psi^{(n)}$ and imposing unit norm on $\boldsymbol{u}$, this formalism leads to a quadratic optimization problem with quadratic constraints that can be solved through eigen decomposition. In Figure 8 (ii.) are shown the amplitude of three different detectors that one can design with the proposed method: corner, symmetric three-fold junction, and T-junction detector. Key points in the image correspond to maxima in the response of the wavelet detector. The optimal steering angle can be determined by root finding, as presented in (Püspöki et al, 2013, Sec. 4.1). The rest of the detection scheme is achieved by traditional techniques which may combine the results at different scales, local maxima search, thresholding, among others. Detections in synthetic and microscopic data is visualized in Figures 8 (iii.), and (iv.), respectively.

The construction presented here makes it possible to capture the local orientation of features in an image within a multiresolution hierarchy (Chenouard and Unser, 2012). The relation between the Riesz transform and steerable filters is studied in (Felsberg and Sommer, 2001). The properties of steerable filters using low-order harmonics are analyzed in 
(Koethe, 2006). The extension of the steerable wavelet design based on the Riesz transform for higher dimensions, along with potential biomedical applications, are presented in (Chenouard and Unser, 2012). Application of steerable wavelets in texture learning for the analysis of CT images are presented in (Depeursinge et al, 2014b, 2015).

\section{Conclusion and Outlook}

We have presented a survey on the directional analysis of bioimages. We have discussed the benefits and drawbacks of classical gradient-based methods, directional multiscale transforms, and multiscale steerable filters. From a user perspective, we have identified steerable wavelets and shearlets as the most attractive methods. They unify high directional selectivity and multiscale analysis, which allows the processing of oriented patterns at different scales. There exist computationally efficient implementations of such schemes that are available for the public. Finally, they are still an active field of research, see the recent papers (Bodmann et al, 2015; Duval-Poo et al, 2015; Kutyniok, 2014; Kutyniok et al, 2014) for shearlets and (Depeursinge et al, 2014a; Dumic et al, 2014; Pad et al, 2014; Schmitter et al, 2014; Ward and Unser, 2014) for steerable filters and wavelets. Furthermore, the corresponding user packages and plugins are maintained and continuously extended.

The state of the art in the field will need to be adjusted to fulfill the upcoming needs of biomedical and biological imaging. Advances in microscopy and in some other measurement systems (CT, $\mathrm{x}$-ray) will shape the future of research. Currently, microscopes are already routinely producing and storing large datasets (often several GBs per measurement) that have to be handled in a fast and efficient way. Moreover, the need to process spatiotemporal data (2D/3D images over time) is becoming unavoidable and is going to require the proper extension of current filter-based schemes. Along with efficiency, the robustness, the precision, and the depth of the extracted information can be improved. Another promising direction of future research is the recovery of directional phase information using complex-valued wavelet transforms such as the monogenic wavelets (Felsberg and Sommer, 2001; Häuser et al, 2014; Heise et al, 2014; Held et al, 2010; Olhede and Metikas, 2009; Soulard et al, 2013; Storath, 2011a; Unser et al, 2009). Preliminary applications include equalization of brightness (Held et al, 2010), detection of salient points (Storath et al, 2015), enhancement of anisotropic structures in fluorescence microscopy (Chenouard and Unser, 2012), and texture segmentation (Storath et al, 2014). Image-analysis tools based on monogenic wavelets are provided by the ImageJ/Fiji plugins Monogenic ${ }^{8}$ and Monogenic Wavelet Toolbox ${ }^{9}$. A further possible direction is the extension of directional wavelet transforms to nonuniform lattices such as polar grids or general graphs (Hammond et al, 2011; Sandryhaila and Moura, 2013; Shuman et al, 2013).

\section{References}

Agam G, Armato S, Wu C (2005) Vessel tree reconstruction in thoracic CT scans with application to nodule detection. IEEE Transactions on Medical Imaging 24(4):486-499

\footnotetext{
${ }^{8}$ Available at http://bigwww.epfl.ch/demo/monogenic/

${ }^{9}$ Available at http://www-m6.ma.tum.de/Mamebia/MonogenicWaveletToolbox/
} 
Aguet F, Geissbühler S, Märki I, Lasser T, Unser M (2009) Super-resolution orientation estimation and localization of fluorescent dipoles using 3-D steerable filters. Optics Express 17(8):6829-6848

Antoine JP, Murenzi R, Vandergheynst P (1999) Directional wavelets revisited: Cauchy wavelets and symmetry detection in patterns. Applied and Computational Harmonic Analysis 6(3):314-345

Bernasconi P, Rust D, Hakim D (2005) Advanced automated solar filament detection and characterization code: Description, performance, and results. Solar Physics 228(12):97-117

Bharath A, Ng J (2005) A steerable complex wavelet construction and its application to image denoising. IEEE Transactions on Image Processing 14(7):948-959

Bigun J (1987) G.H.: Optimal orientation detection of linear symmetry. In: Proceedings of the First IEEE International Conference on Computer Vision, London, Great Britain, pp 433-438

Bigun J, Bigun T, Nilsson K (2004) Recognition by symmetry derivatives and the generalized structure tensor. IEEE Transactions on Pattern Analysis and Machine Intelligence 26(12):1590-1605

Bodmann B, Kutyniok G, Zhuang X (2015) Gabor shearlets. Applied and Computational Harmonic Analysis 38(1):87-114

Candès E, Donoho D (2004) New tight frames of curvelets and optimal representations of objects with piecewise C2 singularities. Communications on Pure and Applied Mathematics 57(2):219-266

Candès E, Donoho D (2005) Continuous curvelet transform: I. Resolution of the wavefront set. Applied and Computational Harmonic Analysis 19(2):162-197

Canny J (1986) A computational approach to edge detection. IEEE Transactions on Pattern Analysis and Machine Intelligence 8(6):679-698

Chaudhury K, Muñoz Barrutia A, Unser M (2010) Fast space-variant elliptical filtering using box splines. IEEE Transactions on Image Processing 19(9):2290-2306

Chenouard N, Unser M (2012) 3D steerable wavelets in practice. IEEE Transactions on Image Processing 21(11):4522-4533

Dan B, Ma AWK, Hároz EH, Kono J, Pasquali M (2012) Nematic-like alignment in SWNT thin films from aqueous colloidal suspensions. Industrial \& Engineering Chemistry Research 51(30):10,232-10,237

Daubechies I (1992) Ten lectures on wavelets, vol 61. SIAM

Daugman J (1985) Uncertainty relation for resolution in space, spatial frequency, and orientation optimized by two-dimensional visual cortical filters. Journal of the Optical Society of America A 2(7):1160-1169

Daugman J (1988) Complete discrete 2D Gabor transforms by neural networks for image analysis and compression. IEEE Transactions on Acoustics, Speech and Signal Processing 36(7):1169-1179

Depeursinge A, Foncubierta-Rodriguez A, Van De Ville D, Müller H (2014a) Rotationcovariant texture learning using steerable Riesz wavelets. IEEE Transactions on Image Processing 23(2):898-908

Depeursinge A, Kurtz C, Beaulieu C, Napel S, Rubin D (2014b) Predicting visual semantic descriptive terms from radiological image data: Preliminary results with liver lesions in CT. IEEE Transactions on Medical Imaging 33(8):1669-1676 
Depeursinge A, Yanagawa M, Leung A, Rubin D (2015) Predicting adenocarcinoma recurrence using computational texture models of nodule components in lung ct. Medical Physics 42(4):2054-2063

Do M, Vetterli M (2005) The contourlet transform: an efficient directional multiresolution image representation. IEEE Transactions on Image Processing 14(12):2091-2106

Dong Y, Tao D, Li X, Ma J, Pu J (2015) Texture classification and retrieval using shearlets and linear regression. IEEE Transactions on Cybernetics 45(3):358-369

Dumic E, Grgic S, Grgic M (2014) IQM2: new image quality measure based on steerable pyramid wavelet transform and structural similarity index. Signal, Image and Video Processing 8(6):1159-1168

Duval-Poo M, Odone F, De Vito E (2015) Edges and corners with shearlets. IEEE Transactions on Image Processing 24(11):3768-3780, DOI 10.1109/TIP.2015.2451175

Felsberg M, Sommer G (2001) The monogenic signal. IEEE Transactions on Signal Processing 49(12):3136-3144

Fonck E, Feigl G, Fasel J, Sage D, Unser M, Rüfenacht D, Stergiopulos N (2008) Effect of ageing on elastin functionality in human cerebral arteries. In: Proceedings of the ASME 2008 Summer Bioengineering Conference (SBC'08), Marco Island FL, USA, pp SBC2008-192,727-1/2

Förstner W (1986) A feature based correspondence algorithm for image matching. International Archives of Photogrammetry and Remote Sensing 26(3):150-166

Frangi A, Niessen W, Vincken K, Viergever M (1998) Multiscale vessel enhancement filtering. In: Medical Image Computing and Computer-Assisted Intervention, Lecture Notes in Computer Science, vol 1496, pp 130-137

Franken E, Duits R (2009) Crossing-preserving coherence-enhancing diffusion on invertible orientation scores. International Journal of Computer Vision 85(3):253-278

Franken E, Duits R, ter Haar Romeny B (2007) Nonlinear diffusion on the 2D Euclidean motion group. In: Scale Space and Variational Methods in Computer Vision, Springer, pp 461-472

Freeman W, Adelson E (1990) Steerable filters for early vision, image analysis, and wavelet decomposition. In: Proceedings of the Third International Conference on Computer Vision, IEEE, pp 406-415

Freeman W, Adelson E (1991) The design and use of steerable filters. IEEE Transactions on Pattern Analysis and Machine Intelligence 13(9):891-906

Freeman WT (1992) Steerable filters and local analysis of image structure. PhD thesis, Massachusetts Institute of Technology

Frikel J (2013) Sparse regularization in limited angle tomography. Applied and Computational Harmonic Analysis 34(1):117-141

Gonzalez G, Fleurety F, Fua P (2009) Learning rotational features for filament detection. In: Proceedings of the IEEE Computer Society Conference on Computer Vision and Pattern Recognition (CVPR'09), IEEE, pp 1582-1589

Grohs P, Kutyniok G (2014) Parabolic molecules. Foundations of Computational Mathematics 14(2):299-337

Guo K, Labate D, Lim WQ (2009) Edge analysis and identification using the continuous shearlet transform. Applied and Computational Harmonic Analysis 27(1):24-46

Hammond D, Vandergheynst P, Gribonval R (2011) Wavelets on graphs via spectral graph theory. Applied and Computational Harmonic Analysis 30(2):129-150

Harris C, Stephens M (1988) A combined corner and edge detector. In: Proceedings of the Fourth Alvey Vision Conference, pp 147-151 
Häuser S, Heise B, Steidl G (2014) Linearized Riesz transform and quasi-monogenic shearlets. International Journal of Wavelets, Multiresolution and Information Processing 12(3)

Heise B, Reinhardt M, Schausberger S, Häuser S, Bernstein S, Stifter D (2014) Fourier plane filtering revisited - analogies in optics and mathematics. Sampling Theory in Signal and Image Processing 13(3):231-248

Held S, Storath M, Massopust P, Forster B (2010) Steerable wavelet frames based on the Riesz transform. IEEE Transactions on Image Processing 19(3):653-667

Honnorat N, Vaillant R, Duncan J, Paragios N (2011) Curvilinear structures extraction in cluttered bioimaging data with discrete optimization methods. In: Proceedings of the Eighth IEEE International Symposium on Biomedical Imaging: From Nano to Macro (ISBI'11), IEEE, pp 1353-1357

Hubel D, Wiesel T (1962) Receptive fields, binocular interaction and functional architecture in the cat's visual cortex. The Journal of Physiology 160(1):106-154

Jacob M, Unser M (2004) Design of steerable filters for feature detection using Canny-like criteria. IEEE Transactions on Pattern Analysis and Machine Intelligence 26(8):10071019

Jacob M, Blu T, Vaillant C, Maddocks J, Unser M (2006) 3-D shape estimation of DNA molecules from stereo cryo-electron micro-graphs using a projection-steerable snake. IEEE Transactions on Image Processing 15(1):214-227

Jacovitti G, Neri A (2000) Multiresolution circular harmonic decomposition. IEEE Transactions on Signal Processing 48(11):3242-3247

Jacques L, Duval L, Chaux C, Peyré G (2011) A panorama on multiscale geometric representations, intertwining spatial, directional and frequency selectivity. Signal Processing 91(12):2699-2730

Jahne B (1997) Digital Image Processing: Concepts, Algorithms, and Scientific Applications, 4th edn. Springer-Verlag New York, Inc., Secaucus, NJ, USA

Jiuxiang H, Razdan A, Femiani J, Ming C, Wonka P (2007) Road network extraction and intersection detection from aerial images by tracking road footprints. IEEE Transactions on Geoscience and Remote Sensing 45(12):4144-4157

Karssemeijer N, te Brake G (1996) Detection of stellate distortions in mammograms. IEEE Transactions on Medical Imaging 15(5):611-619

Kingsbury N (1998) The dual-tree complex wavelet transform: a new technique for shift invariance and directional filters. In: IEEE Digital Signal Processing Workshop, vol 86, pp 120-131

Koethe U (2006) Low-level feature detection using the boundary tensor. In: Weickert J, Hagen $\mathrm{H}$ (eds) Visualization and Processing of Tensor Fields, Mathematics and Visualization, Springer Berlin Heidelberg, pp 63-79

Köthe U (2003) Edge and junction detection with an improved structure tensor. In: Pattern Recognition, Lecture Notes in Computer Science, vol 2781, Springer, pp 25-32

Kutyniok G (2012) Data separation by sparse representations. In: Compressed Sensing: Theory and Applications, Cambridge University Press

Kutyniok G (2014) Geometric separation by single-pass alternating thresholding. Applied and Computational Harmonic Analysis 36(1):23-50

Kutyniok G, Labate D (2009) Resolution of the wavefront set using continuous shearlets. Transactions of the American Mathematical Society 361(5):2719-2754

Kutyniok G, Labate D (2012) Shearlets: Multiscale analysis for multivariate data. Birkhauser Basel 
Kutyniok G, Lim WQ, Reisenhofer R (2014) Shearlab 3D: Faithful digital shearlet transforms based on compactly supported shearlets. arXiv preprint arXiv:14025670

Labate D, Lim WQ, Kutyniok G, Weiss G (2005) Sparse multidimensional representation using shearlets. In: Optics \& Photonics 2005, pp 59,140U-59,140U

Lam B, Gao Y, Liew AC (2010) General retinal vessel segmentation using regularizationbased multiconcavity modeling. IEEE Transactions on Medical Imaging 29(7):13691381

Larkin K (2001) Natural demodulation of two-dimensional fringe patterns. II. stationary phase analysis of the spiral phase quadrature transform. Journal of the Optical Society of America A 18:1871-1881

Larkin K, Bone DJ, Oldfield MA (2001) Natural demodulation of two-dimensional fringe patterns. I. general background of the spiral phase quadrature transform. Journal of the Optical Society of America A 18:1862-1870

Lee T (1996) Image representation using 2D Gabor wavelets. IEEE Transactions on Pattern Analysis and Machine Intelligence 18(10):959-971

Lorenz C, Carlsen IC, Buzug T, Fassnacht C, Weese J (1997) Multi-scale line segmentation with automatic estimation of width, contrast and tangential direction in 2D and 3D medical images. In: Proceedings of the First Joint Conference Computer Vision, Virtual Reality and Robotics in Medicine and Medical Robotics and Computer-Assisted Surgery CVRMed - MRCAS'97, Springer, pp 233-242

Ma J, Plonka G (2010) The curvelet transform. IEEE Signal Processing Magazine 27(2):118133

Mallat S (2008) A wavelet tour of signal processing: the sparse way. Academic Press

Marr D, Hildreth E (1980) Theory of edge detection. Proceedings of the Royal Society of London Series B, Biological Sciences 207(1167):187-217

Meijering E (2010) Neuron tracing in perspective. Cytometry A 77(7):693-704

Meijering E, Jacob M, Sarria JC, Steiner P, Hirling H, Unser M (2004) Design and validation of a tool for neurite tracing and analysis in fluorescence microscopy images. Cytometry Part A 58A(2):167-176

Olhede S, Metikas G (2009) The monogenic wavelet transform. IEEE Transactions on Signal Processing 57(9):3426-3441

Olshausen B, Field D (1996) Emergence of simple-cell receptive field properties by learning a sparse code for natural images. Nature 381:607-609

Pad P, Uhlmann V, Unser M (2014) VOW: Variance-optimal wavelets for the steerable pyramid. In: IEEE International Conference on Image Processing (ICIP), pp 2973-2977

Patton N, Aslam T, MacGillivray T, Deary I, Dhillon B, Eikelboom R, Yogesan K, Constable I (2006) Retinal image analysis: concepts, applications and potential. Progress in Retinal and Eye Research 25(1):99-127

Perona P (1992) Steerable-scalable kernels for edge detection and junction analysis. Image and Vision Computing 10(10):663-672

Portilla J, Simoncelli E (2000) A parametric texture model based on joint statistics of complex wavelet coefficients. International Journal of Computer Vision 40(1):49-70

Püspöki Z, Unser M (2015) Template-free wavelet-based detection of local symmetries. IEEE Transactions on Image Processing 24(10):3009-3018

Püspöki Z, Vonesch C, Unser M (2013) Detection of symmetric junctions in biological images using 2-D steerable wavelet transforms. In: Proceedings of the Tenth IEEE International Symposium on Biomedical Imaging: From Nano to Macro (ISBI'13), San Francisco CA, USA, pp 1488-1491 
Rezakhaniha R, Agianniotis A, Schrauwen J, Griffa A, Sage D, Bouten C, van de Vosse F, Unser M, Stergiopulos N (2012) Experimental investigation of collagen waviness and orientation in the arterial adventitia using confocal laser scanning microscopy. Biomechanics and Modeling in Mechanobiology 11(3-4):461-473

Sandryhaila A, Moura J (2013) Discrete signal processing on graphs. IEEE Transactions on Signal Processing 61(7):1644-1656

Sato Y, Nakajima S, Shiraga N, Atsumi H, Yoshida S, Koller T, Gerig G, Kikinis R (1998) Three-dimensional multi-scale line filter for segmentation and visualization of curvilinear structures in medical images. Medical Image Analysis 2(2):143-168

Schmitter D, Delgado-Gonzalo R, Krueger G, Unser M (2014) Atlas-free brain segmentation in 3D proton-density-like MRI images. In: Proceedings of the IEEE International Symposium on Biomedical Imaging: From Nano to Macro (ISBI), pp 629-632

Schuh M, Banda J, Bernasconi P, Angryk R, Martens P (2014) A comparative evaluation of automated solar filament detection. Solar Physics 289(7):2503-2524

Selesnick I, Baraniuk R, Kingsbury N (2005) The dual-tree complex wavelet transform. IEEE Signal Processing Magazine 22(6):123-151

Semler L, Dettori L (2006) Curvelet-based texture classification of tissues in computed tomography. In: Proceedings of the Thirteenth IEEE International Conference on Image Processing (ICIP'06), IEEE, pp 2165-2168

Sherlock B, Monro D, Millard K (1994) Fingerprint enhancement by directional Fourier filtering. In: IEE Proceedings on Vision, Image and Signal Processing, vol 141, pp 87-94

Shuman D, Narang S, Frossard P, Ortega A, Vandergheynst P (2013) The emerging field of signal processing on graphs: Extending high-dimensional data analysis to networks and other irregular domains. IEEE Signal Processing Magazine 30(3):83-98

Simoncelli E, Farid H (1996) Steerable wedge filters for local orientation analysis. IEEE Transactions on Image Processing 5(9):1377-1382

Simoncelli E, WT F (1995) The steerable pyramid: A flexible architecture for multi-scale derivative computation. In: Proceedings of the Second IEEE International Conference on Image Processing (ICIP'95), IEEE, vol 3, pp 444-447

Soulard R, Carre P, Fernandez-Maloigne C (2013) Vector extension of monogenic wavelets for geometric representation of color images. IEEE Transactions on Image Processing 22(3):1070-1083

Starck JL, Candès E, Donoho D (2002) The curvelet transform for image denoising. IEEE Transactions on Image Processing 11(6):670-684

Starck JL, Murtagh F, Candes E, Donoho D (2003) Gray and color image contrast enhancement by the curvelet transform. IEEE Transactions on Image Processing 12(6):706-717

Storath M (2011a) Directional multiscale amplitude and phase decomposition by the monogenic curvelet transform. SIAM Journal on Imaging Sciences 4(1):57-78

Storath M (2011b) Separation of edges in X-Ray images by microlocal analysis. Proceedings in Applied Mathematics and Mechanics (PAMM) 11(1):867-868

Storath M, Weinmann A, Unser M (2014) Unsupervised texture segmentation using monogenic curvelets and the Potts model. In: IEEE International Conference on Image Processing (ICIP), pp 4348-4352

Storath M, Demaret L, Massopust P (2015) Signal analysis based on complex wavelet signs. Applied and Computational Harmonic Analysis In press, preprint arXiv:1208.4578

Tupin F, Maitre H, Mangin JF, Nicolas JM, Pechersky E (1998) Detection of linear features in SAR images: application to road network extraction 
Unser M, Chenouard N (2013) A unifying parametric framework for 2D steerable wavelet transforms. SIAM Journal on Imaging Sciences 6(1):102-135

Unser M, Van De Ville D (2010) Wavelet steerability and the higher-order Riesz transform. IEEE Transactions on Image Processing 19(3):636-652

Unser M, Sage D, Van De Ville D (2009) Multiresolution monogenic signal analysis using the Riesz-Laplace wavelet transform. IEEE Transactions on Image Processing 18(11):2402-2418

Vandergheynst P, Gobbers JF (2002) Directional dyadic wavelet transforms: Design and algorithms. IEEE Transactions on Image Processing 11(4):363-372

Ward J, Unser M (2014) Harmonic singular integrals and steerable wavelets in $l_{2}\left({ }^{d}\right)$. Applied and Computational Harmonic Analysis 36(2):183-197

Ward J, Chaudhury K, Unser M (2013) Decay properties of Riesz transforms and steerable wavelets. SIAM Journal on Imaging Sciences 6(2):984-998

Yi S, Labate D, Easley G, Krim H (2009) A shearlet approach to edge analysis and detection. IEEE Transactions on Image Processing 18(5):929-941

Yuan Y, Shih F, Jing J, Wang H, Chae J (2011) Automatic solar filament segmentation and characterization. Solar Physics 272(1):101-117 